\title{
Automatic Removal of Impulse Noise from Highly Corrupted Images
}

\author{
Vitaly Kober ${ }^{1}$, Mikhail Mozerov ${ }^{2}$, and Josué Álvarez-Borrego ${ }^{3}$ \\ ${ }^{1}$ Department of Computer Science, Division of Applied Physics, \\ CICESE, Ensenada, B.C. 22860, Mexico \\ vkober@cicese.mx \\ ${ }^{2}$ Laboratory of Digital Optics, Institute for Information Transmission Problems, \\ Bolshoi Karetnii 19, 101447 Moscow, Russia \\ mozer@itp.ru \\ ${ }^{3}$ Dirección de Telemática, \\ CICESE, Ensenada, B.C. 22860, Mexico \\ josuedcicese.mx
}

\begin{abstract}
An effective algorithm for automatic removal impulse noise from highly corrupted monochromatic images is proposed. The method consists of two steps. Outliers are first detected using local spatial relationships between image pixels. Then the detected noise pixels are replaced with the output of a rank-order filter over a local spatially connected area excluding the outliers, while noise-free pixels are left unaltered. Simulation results in test images show a superior performance of the proposed filtering algorithm comparing with conventional filters. The comparisons are made using mean square error, mean absolute error, and subjective human visual error criterion.
\end{abstract}

\section{Introduction}

Digital images are often corrupted by impulse noise due to a noise sensor or channel transmission errors. The major objective of impulse noise removal is to suppress the noise while preserving the image details. Various algorithms have been proposed for impulse noise removal [1-5]. Basically the most of these algorithms are based on the calculation of rank-order statistics [6]. If filters are implemented uniformly across an image then they tend to modify pixels that are undisturbed by noise. Moreover, they are prone to edge jitter when the percentage of impulse noise is large. Consequently, suppression of impulses is often at expense of blurred and distorted features. Effective techniques usually consist of two steps. First a filter detects corrupted pixels and then a noise cancellation scheme is applied only to detected noisy pixels. Recently nonlinear filters for monochrome images with a signal-dependent shape of the moving window have been proposed [7]. In this paper, we extend this approach to automatic suppressing the impulse noise in highly corrupted images. First outliers are detected using local spatial relationships between image pixels. Then the detected noise pixels are replaced with the output of an appropriate rank-order filter computed over a local spatially connected area excluding the outliers from the area. In the case of 
independent impulse noise, the proposed detector greatly reduces the miss probability of impulse noise. The performance of the proposed filter is compared with that of conventional algorithms.

The presentation is organized as follows. In Section 2, we present a new efficient algorithm for automatic detection of noise impulses. A modified filtering algorithm using the proposed detector is also described. In Section 3, with the help of computer simulation we test the performance of the conventional and proposed filters. Section 4 summarizes our conclusions.

\section{Automatic Detection and Removal Impulse Noise}

In impulse noise models, corrupted pixels are often replaced with values near to the maximum and minimum of the dynamic range of a signal. In our experiments, we consider a similar model in which a noisy pixel can take a random value either from sub-ranges of the maximum or the minimum values with a given probability. The distribution of impulse noise in the sub-ranges can be arbitrary. To detect impulse noise in an image, we use the concept of a spatially connected neighborhood ( $S C N)$. An underlying assumption is as follows: image pixels geometrically close to each other belong to the same structure or detail. The spatially connected neighborhood is defined as a subset of pixels $\left\{v_{n, m}\right\}$ of a moving window, which are spatially connected with the central pixel of the window, and whose values deviate from the value of the central pixel $v_{k, l}$ at most predetermined quantities $-\varepsilon_{v}$ and $+\varepsilon_{v}$ [7]:

$$
\operatorname{SCN}\left(v_{k, l}\right)=\operatorname{CON}\left(\left\{v_{n, m}: v_{k, l}-\varepsilon_{v} \leq v_{n, m} \leq v_{k, l}+\varepsilon_{v}\right\}\right),
$$

where $\operatorname{CON}(X)$ denotes four- or eight-connected region including the central pixel of the moving window. The size and shape of a spatially connected neighborhood are dependent on characteristics of image data and on parameters, which define measures of homogeneity of pixel sets. So the spatially connected neighborhood is a spatially connected region constructed for each pixel, and it consists of all the spatially connected pixels, which satisfy a property of similarity with the central pixel.

We assume that the size of the $S C N$ of a noise cluster is relatively small comparing to that of details of image to be processed. Therefore impulsive noise can be detected by checking the size of the cluster; that is, if $S \leq \mathrm{M}$ then the impulse is detected. Here $S=\operatorname{SIZE}(S C N)$ is the number of pixels included in the $S C N$ constructed around the central pixel of the moving window with the parameter $\mathcal{E}_{v}$ for adjacent pixels, $M$ is a given threshold value for detection of noise clusters. Actually the detection depends on two integer parameters; that is, $\mathcal{E}_{v}$ and $M$. Extensive computer simulations have shown that the best value of $M$, which yields minimum detection errors of noise clusters for various noise distributions, can be expressed as a function of a given noise distribution and a chosen value of $\varepsilon_{v}$. Let us consider model of impulsive noise. A test gray scale image has $Q=256$ quantization levels and $N$ pixels. The probability of independent corruption of image pixels by impulse noise at the level $q$ is equal to $P(q)$ $(0 \leq q \leq Q-1)$. The probability of noise impulse occurring can be calculated as

$$
p=\sum_{q=0}^{Q-1} P(q),
$$


and the expected number of impulses in the image is given by

$$
N_{\text {imp }}=p N .
$$

For the considering detector, if the absolute difference between the noise impulse and pixels of neighborhood is less or equal to a chosen value of $\varepsilon_{v}$ then the impulse is invisible for the detector. Therefore the total number of detectable impulses is less than $N_{i m p}$ in Eq. (3). In this case the expected number of outliers is given by

$$
\tilde{N}_{i m p}=N \sum_{q=0}^{Q-1} \tilde{P}(q),
$$

where $\tilde{P}(q)$ is the probability of detection of an impulse at the level $q$. If the distribution of the image signal is spatially homogeneous then the probability of noise impulse detection can be approximately estimated with the help of the histogram of uncorrupted test image,

$$
\tilde{P}(q) \approx P(q)\left(1-\sum_{l=0}^{Q-1} h_{l}\left[|l-q| \leq \varepsilon_{v}\right] / N\right),
$$

where $\left\{h_{q}\right\}$ is the histogram of uncorrupted image, [.] denotes the following function: 1 , if the statement in brackets is true and 0 , otherwise.

Since the histogram of the uncorrupted image is usually inaccessible then the estimation of this histogram can be written as

$$
h_{q}=\frac{\tilde{h}_{q}-N P(q)}{1-p},
$$

where $\left\{\tilde{h}_{q}\right\}$ is the available histogram of the observed noisy image.

The proposed detector of impulse noise takes into account the size of the SCN. Now we know how many impulses can be detected by the detector. Obviously, such detector omits impulses with the size greater than $M$. The probability $\operatorname{Pr}(\mathrm{M})$ of occurrence of four-connected noise clusters of the size $M$ can be computed using the formulas given in the papers $[8,9]$. In this way the expected number and the probability of occurrence of all clusters of the size greater than $M$ can be obtained. We can state that if the expected number of clusters of the size greater than $M$ (for a given image and a noise distribution) is less than unity then the value of the threshold $M$ is optimal. Formally the statement can be written as

$$
M=S \text { if } N_{\text {imp }>S}=N_{\text {imp }}-N \sum_{m=1}^{S} \operatorname{Pr}(m)<1 \leq N_{i m p}-N \sum_{m=1}^{S-1} \operatorname{Pr}(m)
$$

where $N_{\text {imp }>S}$ is the expected number of clusters of the size greater than $S$ pixels.

The probability of occurrence of a four-connected noise cluster of the size $M$ in a moving window can be computed using the addition formula of probabilities. The noise cluster occurs simultaneously with one of the mutually exclusive events $H_{1}, \ldots$, $H_{N}$. Here $H_{k}$ is the event denoting that there is a noise cluster of the size exactly $M$ 
noise impulses surrounded by uncorrupted image pixels. The probability of occurrence of a noise cluster of the size $M$ at a given image pixel is given as $[8,9]$

$$
\operatorname{Pr}(M)=\sum_{k=1}^{N} \operatorname{Pr}\left(H_{k}\right),
$$

where the probability of the event $H_{k}$ is $\operatorname{Pr}\left(H_{k}\right)=P^{M}(1-P)^{E_{k}(M)}, E_{k}(M)$ is the number of surrounded uncorrupted image pixels. Taking into account that some of the probabilities $\operatorname{Pr}\left(H_{k}\right)$ are equal, the Eq.(8) is computationally simplified to

$$
\operatorname{Pr}(M)=p^{M} \sum_{k=1}^{K(M)} C_{k}(M)(1-p)^{E_{k}(M)},
$$

where $K(M)$ is the number of groups, each of them contains $C_{k}(M)$ events $H_{k}$ with the equal probabilities $\operatorname{Pr}\left(H_{k}\right), \mathrm{k}=1, \ldots K(M) . C_{k}(M), E_{k}(M)$ are coefficients determined from the geometry (binary region of support) of the cluster of noise. For example, the number of groups with $M=2$ is $K(2)=1$, and the number of surrounding fourconnected uncorrupted pixels is $E_{l}(M)=6$. The number of the events is $C_{l}(M)=4$ (four possible variants of the noise cluster on the grid including the given pixel). These coefficients are provided in Table 1.

Table 1. Coefficients for calculating the probability of impulsive clusters

\begin{tabular}{|c|c|c|c|c|}
\hline $\begin{array}{c}\text { Size of } \\
\text { cluster M }\end{array}$ & $K(M)$ & $k$ & $C_{k}(M)$ & $E_{k}(M)$ \\
\hline 1 & 1 & 1 & 1 & 4 \\
\hline 2 & 1 & 1 & 4 & 6 \\
\hline 3 & 2 & 1 & 12 & 7 \\
& & 2 & 6 & 8 \\
\hline 4 & 3 & 1 & 36 & 8 \\
& & 3 & 32 & 9 \\
& 5 & 1 & 8 & 10 \\
\hline 5 & & 2 & 5 & 8 \\
& & 3 & 100 & 9 \\
& & 4 & 60 & 10 \\
& & 5 & 10 & 11 \\
& & & & 12 \\
\hline
\end{tabular}

With the help of Table 1 and Eq. (9), the probability of occurrence of a fourconnected impulse noise cluster of the size $M$ can be easily calculated. Table 2 presents the probability of occurrence of impulse cluster of size $M$ versus the probability of impulse noise on a rectangular grid. We see that when the probability of impulse noise is high, the occurrence of impulse cluster is very likely. 
Table 2. The probability of occurrence of impulse clusters of the size $M$ versus the probability $p$ of impulse noise

\begin{tabular}{|c|c|c|c|}
\hline \multirow{2}{*}{$\mathrm{M}$} & \multicolumn{3}{|c|}{ Probability of impulse noise } \\
\cline { 2 - 4 } & $p=0.01$ & $p=0.1$ & $p=0.2$ \\
\hline 0 & 0.99 & 0.9 & 0.8 \\
\hline 1 & $5.6 \times 10^{-3}$ & $6.5 \times 10^{-2}$ & $8.2 \times 10^{-2}$ \\
\hline 2 & $3.7 \times 10^{-4}$ & $2.1 \times 10^{-2}$ & $4.2 \times 10^{-2}$ \\
\hline 3 & $1.7 \times 10^{-5}$ & $8.3 \times 10^{-3}$ & $2.8 \times 10^{-2}$ \\
\hline 4 & $7 \times 10^{-7}$ & $3 \times 10^{-3}$ & $1.8 \times 10^{-2}$ \\
\hline 5 & $2.8 \times 10^{-8}$ & $1.1 \times 10^{-3}$ & $1.1 \times 10^{-2}$ \\
\hline
\end{tabular}

Finally the proposed algorithm of impulse noise detection consists of the following steps.

- $\quad$ Choose two initial values for $\varepsilon_{v} \in[1,(\mathrm{Q}-1)]$, say $\varepsilon_{v}$ max and $\mathcal{E}_{v}$ min, and then calculate $\varepsilon_{v}=\left(\varepsilon_{v \max }+\varepsilon_{v \min }\right) / 2$.

- $\quad$ Compute $\tilde{N}_{i m p}$ and $M$ using Eqs. (4)-(7), noise distribution and threshold $\varepsilon_{v}$.

- $\quad$ Form the $S C N$ with $\varepsilon_{v}$ and calculate the number of detected impulses, say $D$.

- $\quad$ Compare $D$ with $\tilde{N}_{i m p}$, and if $\left(D=\tilde{N}_{i m p}\right.$ or $\varepsilon_{v}=\varepsilon_{v \max }$ or $\left.\varepsilon_{v}=\varepsilon_{v \min }\right)$ then the optimal pair of $\varepsilon_{v}$ and $M$ is found, else go to the next step.

- If $D>\tilde{N}_{i m p}$ then set $\varepsilon_{v \text { min }}=\varepsilon_{v}$, else set $\varepsilon_{v \max }=\varepsilon_{v}$. Calculate $\varepsilon_{v}=\left(\varepsilon_{v \max }+\varepsilon_{v \min }\right) / 2$ and go to the second step.

Computer experiments with test images corrupted by various kinds of impulse noise have showed that the integer function $D\left(\varepsilon_{v}\right)$ is monotonically decreasing. Thus the solution of the proposed iterative algorithm with respect to $\varepsilon_{v}$ is unique. Since $\varepsilon_{v \text { max }}$, $\varepsilon_{v \min }$, and $\varepsilon_{v}$ are integer then the number of iterations for $Q=256$ is limited by 7 .

When the map of detected impulses with the calculated parameters is obtained, the noisy pixels are replaced with the output of any appropriate filter. In our case the median value of at least 3 uncorrupted neighboring pixels is used.

\section{Computer Experiments}

Signal processing of an image degraded due to impulse noise is of interest in a variety of tasks. Computer experiments are carried out to illustrate and compare the performance of conventional and proposed algorithms. In this paper, we will base our comparisons on the mean square error (MSE), the mean absolute error (MAE), and a subjective visual criterion. The empirical normalized mean square error is given by

$$
M S E=\frac{\sum_{n=1}^{N_{x}} \sum_{m=1}^{M_{y}}\left|v_{n, m}-\hat{v}_{n, m}\right|^{2}}{\sum_{n=1}^{N_{x}} \sum_{m=1}^{M_{y}} v_{n, m}{ }^{2}},
$$


where $\left\{v_{n, m}\right\}$ and $\left\{\hat{v}_{n, m}\right\}$ are the original image and its estimate (filtered image), respectively. In our simulations, $N_{x}=256, M_{y}=256$ (256x256 image resolution), and each pixel has 256 levels of quantization. The empirical normalized mean absolute error is defined as

$$
M A E=\frac{\sum_{n=1}^{N_{x}} \sum_{m=1}^{M_{y}}\left|v_{n, m, k}-\hat{v}_{n, m, k}\right|}{\sum_{n=1}^{N_{x}} \sum_{m=1}^{M_{y}}\left|v_{n, m, k}\right|} .
$$

The use of these error measures allows us to compare the performance of each filter. Fig. 1 shows a test image. The test image degraded due to impulsive noise is shown in Fig. 2.

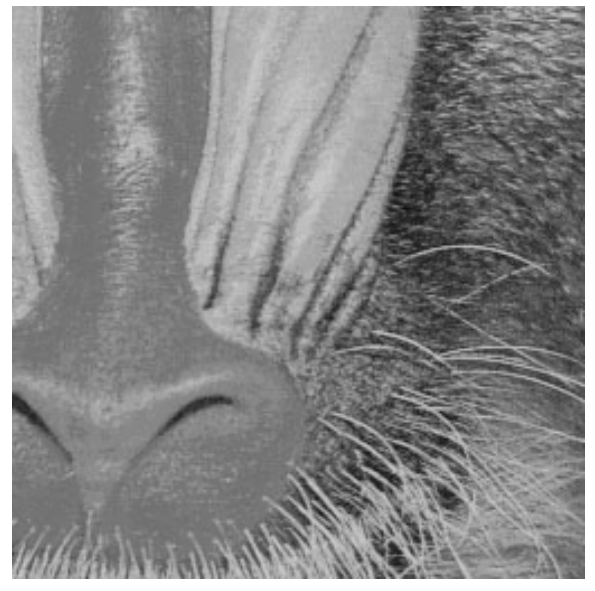

Fig. 1. Original image

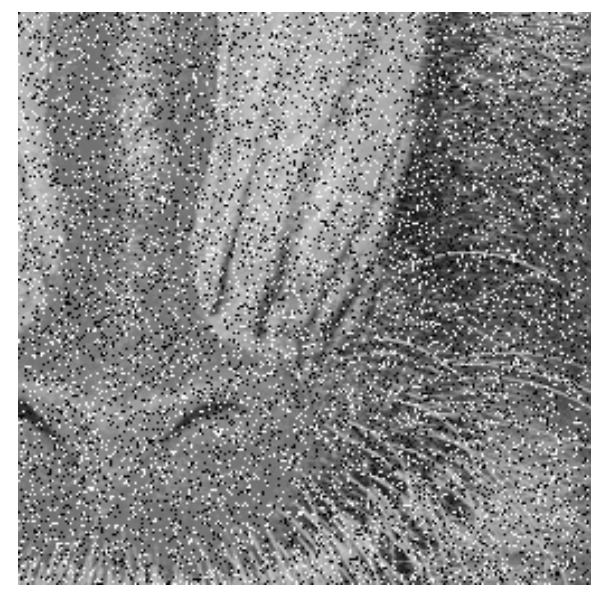

Fig. 2. Noisy image

The probability of independent noise impulse occurrence is 0.2 . In computer simulation, the values of impulses were set to $0-15$ or 240-255 with equal probability. Table 3 shows the errors under the MSE and MAE criteria for the median filter (MED) of 3x3 pixels, fuzzy technique (FF) [5], and the proposed filter.

Table 3. Impulse noise suppression with different filters

\begin{tabular}{|c|c|c|}
\hline \multirow{2}{*}{ Type of Filters } & \multicolumn{2}{|c|}{ Measured Errors } \\
\cline { 2 - 3 } & MSE & MAE \\
\hline Noisy image & 0.17 & 0.162 \\
\hline MED 3x3 & 0.065 & 0.012 \\
\hline FF algorithm & 0.023 & 0.009 \\
\hline Proposed algorithm & 0.019 & 0.005 \\
\hline
\end{tabular}

The parameters $M$ and $\varepsilon_{v}$ are automatically calculated with the proposed algorithm described in Section 2. We see that in this case the proposed filter has the best 
performance with respect to the MSE and MAE. Now we carry out visual comparison of the filtering results with the median and the proposed filters. Figures 3 and 4 show the filtered images obtained from the noisy image with the median filter and the proposed filter, respectively. The proposed filter using the spatial pixel connectivity has a strong ability in impulse noise suppression and a very good preservation of fine structures and details. The visual comparison shows that the filtered image with the median filter is much smoother than the output image after filtering with proposed method.

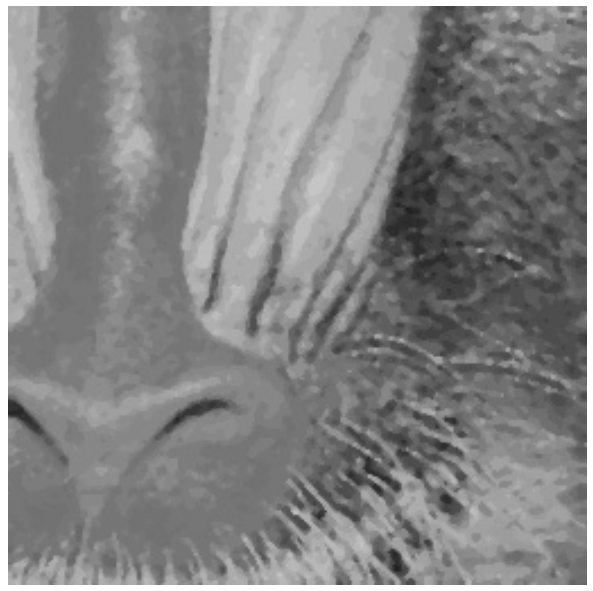

Fig. 3. Filtered image by MED filter

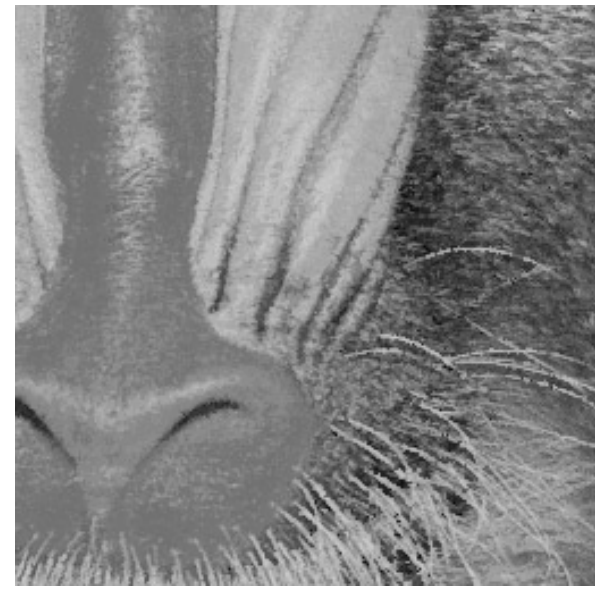

Fig. 4. Filtered image by the proposed method

\section{Conclusion}

In this paper, we have presented a new algorithm for automatic detection and suppression of impulse noise in highly corrupted images. The filter utilizes an explicit use of spatial relations between image elements. When the input image is degraded due impulse noise, extensive testing has shown that the proposed spatially adaptive filter outperforms conventional filters in terms of the mean square error, the mean absolute error, and the subjective visual criterion.

\section{References}

1. Pitas I. and Venetsanopoulos A.N., Nonlinear digital filters. Principles and applications, Kluwer Academic Publishers, Boston (1990).

2. Tsekeridou S., Kotropoulos C., Pitas I., Adaptive order statistic filters for the removal of noise from corrupted images, Optical Engineering, Vol. 37, (1998), 2798-2815.

3. Abreu E., Linghtstone M., Mitra S.K., and Arakawa K., A new efficient approach for the removal of impulse noise from highly corrupted images, IEEE Trans. on Image Processing, Vol. 2, No. 6, (1993), 1012-1025. 
4. Lehmann T., Oberschelp W., Pelikan E., Repges R., Image processing for medical images, Springer-Verlag, Berlin, Heidelberg, New York (1997).

5. Zhang D. and Wang Z., Impulse noise detection and removal using fuzzy techniques, Electronics Letter, Vol. 33, No. 5, (1997), 378-379.

6. David H.A., Order statistics, Wiley, New York (1970).

7. Kober V., Mozerov M., Alvarez-Borrego J., Nonlinear filters with spatially connected neighborhoods, Optical Engineering, Vol. 40, No. 6, (2001), 971-983.

8. Mozerov M., Kober V., Choi T., Noise Removal from highly corrupted color images with adaptive neighborhoods, IEICE Trans. on Fund., Vol. E86-A, No. 10, 2003, 2713-2717.

9. Kober V., Mozerov M., Alvarez-Borrego J., Spatially adaptive algorithms for impulse noise removal from color images, Lecture Notes in Computer Science, Vol. 2905, (2003), 113-120. 